\title{
Stability of the Infrared Array Camera for the Spitzer Space Telescope
}

S. Carey, J. Surace, M. Lacy, W. Glaccum, P. Lowrance, et al.

S. Carey, J. Surace, M. Lacy, W. Glaccum, P. Lowrance, J. L. Hora, S. Willner, "Stability of the Infrared Array Camera for the Spitzer Space Telescope," Proc. SPIE 7010, Space Telescopes and Instrumentation 2008: Optical, Infrared, and Millimeter, 70102V (12 July 2008); doi: $10.1117 / 12.790030$

SPIE Event: SPIE Astronomical Telescopes + Instrumentation, 2008, Marseille, France 


\title{
Stability of the Infrared Array Camera for the Spitzer Space Telescope
}

\author{
S. Carey*a, J. Surace, M. Lacy ${ }^{\mathrm{a}}$, W. Glaccum ${ }^{\mathrm{a}}$, P. Lowrance ${ }^{\mathrm{a}}$ J. L. Hora ${ }^{\mathrm{b}}$, S. Willner, ${ }^{\mathrm{b}}$ \\ ${ }^{a}$ Spitzer Science Center, MS 220-6, California Institute of Technology, Pasadena, CA, USA, 91125; \\ ${ }^{\mathrm{b}}$ Harvard-Smithsonian Center for Astrophysics, 60 Garden Street, Cambridge, MA, USA, 02138
}

\begin{abstract}
We present an analysis of the stability of the Infrared Array Camera (IRAC) on board the Spitzer Space Telescope over the first 4.5 years of in-flight operations. IRAC consists of two InSb and two Si:As 256x256 imaging arrays with passbands centered on 3.6, 4.5. 5.8 and 8.0 microns. Variations in photometric stability, read noise, dark offsets, pixel responsivity and number of hot and noisy pixels for each detector array are trended with time. To within our measurement uncertainty, the performance of the IRAC arrays has not changed with time. The most significant variation is that number of hot pixels in the 8 micron array has increased linearly with time at a rate of 60 pixels per year. We expect that the 3.6 and 4.5 micron arrays should remain stable during the post-cryogenic phase of the Spitzer mission. We will briefly discuss some science that is enabled by the excellent stability of IRAC.
\end{abstract}

Keywords: infrared detectors, space telescopes, Spitzer Space Telescope, IRAC

\section{INTRODUCTION}

The Infrared Array Camera ${ }^{1,2}$ (IRAC) is one of the three science instruments aboard the Spitzer Space Telescope ${ }^{3}$. IRAC consists of four $256 \times 256$ arrays viewing two sets of $5.2 \times 5.2$ arcminute fields of view with broadband filters spanning 3-9 $\mu \mathrm{m}$. The fields of view are slightly offset with a gap of $\sim 1$ arcminute between them with two pickoff mirrors feeding the IRAC optical system. Each field of view is imaged by one InSb array and one Si:As impurity band conduction (IBC) array. Light from each pickoff mirror is re-imaged using a $\mathrm{MgF}_{2}-\mathrm{ZnS}$ doublet lens and a Ge beamsplitter reflects the light to the short wavelength arrays and transmits to the longer wavelength arrays. The passbands for the two fields of view are centered on $3.6 / 5.8 \mu \mathrm{m}$ and $4.5 / 8.0 \mu \mathrm{m}$, respectively. The platescale for each array is $\sim 1.2$ arcseconds. As Spitzer is diffraction limited only for wavelengths greater than $5 \mu \mathrm{m}$, the InSb arrays are undersampled. Optical distortion for the camera is of order $1 \%$.

IRAC consists of two components, a warm electronic section which contains the control electronics for IRAC including the digital signal processing of the images and allows selection of the four programmable biases and array temperature set points. The warm electronics section self-heats to temperatures of $\sim 20^{\circ} \mathrm{C}$. The cold assembly is contained in Spitzer's multiple instrument chamber and is cooled by superfluid He vapor from the cryostat to $\sim 1.3 \mathrm{~K}$. The cold assembly contains the IRAC focal planes, optics, calibration lamps and focal plane heaters. The cold assembly is well isolated from the warm electronics with minimal thermal coupling between. The IRAC focal planes are actively thermally controlled with temperatures stable to a milli-Kelvin. This stability is achieved by heating the arrays to their operating temperatures of $15 \mathrm{~K}$ for the InSb and $6 \mathrm{~K}$ for the Si:As arrays using an active feedback thermal control via Cernox sensors. The arrays are read out using a set of four multiplexers for each channel. Each multiplexer reads one column at a time with four columns addressed at once. The arrays are read using non-destructive pairs of Fowler samples with the measured value being the average of the difference of the signal and pedestal pairs. IRAC has two base modes of operation, full array where the entire array is sampled with a readout time of 0.2 seconds and subarray mode. In subarray mode, a $32 \times 32$ portion of each array is readout with a sampling time of 0.01 seconds. The subarray mode was designed to extend the dynamic range of IRAC for bright sources as well as facilitate studies of time varying signals.

IRAC has been designed with the ability of very deep, confusion limited imaging to probe the high redshift universe and very fast mapping to perform studies of debris disks and Galactic star formation as well as conduct large area extragalactic surveys. It has a unique combination of sensitivity and large field of view. For the longest integration time used, 100 seconds, IRAC has one sigma point source sensitivities of $0.6,1.2,8.0$ and $9.8 \mu \mathrm{Jy}$. IRAC is operated with a single astronomical observation template with a moderate number of observer defined parameters including a set of six

Space Telescopes and Instrumentation 2008: Optical, Infrared, and Millimeter, edited by Jacobus M. Oschmann, Jr., Mattheus W. M. de Graauw, Howard A. MacEwen, Proc. of SPIE Vol. 7010, 70102V, (2008) · 0277-786X/08/\$18 - doi: 10.1117/12.790030 
integration times for full array mode and three integration times for subarray ${ }^{4}$. A set of mapping and dithering strategies are also available as well as hybrid modes using multiple frame times to provide high dynamic range observations and maximize signal-to-noise for sources with stellar-like spectral energy distributions. IRAC has met and exceeded its design goals for photometric accuracy with an absolute photometric accuracy of better than $3 \%$ in all bands and is currently most limited by the $1.5 \%$ error in absolute flux scaling ${ }^{5}$ (limited by our knowledge of the true fluxes of the fundamental calibrators).

IRAC has performed flawlessly for over five years. Despite several significant solar events, the detectors have not degraded appreciably over time. Among science highlights from the instrument, it has mapped $>130$ square degrees of the Galactic plane ${ }^{6}$, detected galaxies out to $\mathrm{z}=6.7^{7}$, and made the first detection of thermal emission from a planet around another $\operatorname{star}^{8}$.

In general, IRAC is operated in campaigns of duration of one to two weeks with about one month spacing between campaigns. The instrument is powered on at the beginning and powered off at the end of each campaign. The campaigns consist of science observations and instrumental calibration activities to support those observations. As the IRAC shutter is not used in flight, we employ a shutterless calibration strategy which involved making detector bias measurements called skydarks using low background, low source density fields and detector gain (flat-field) measurements against bright background regions (the ecliptic plane). The skydarks and flat-field (skyflat) observations are executed at the beginning and end of each IRAC campaign. In addition to the bias and gain calibrations, periodic observations of primary flux calibrators are done at the beginning and end of each campaign. The calibrators are a well characterized set of seven A0 dwarfs and K0-K2 giants. Photometric stability during the campaign is checked by observations of one of a set of secondary calibrators once per observation period between data downlinks. Downlinks typically occur every 12 or 24 hours depending on data volume, and the secondary calibrators are in the ecliptic plane to minimize the amount of slewing for the calibration. In addition to the calibration activities, the flood lamps are exercised at the end of every campaign to provide a secondary check for dead and hot pixels.

To mitigate long-term persistent images at 3.6 and $8.0 \mu \mathrm{m}$, the detector arrays are thermally annealed ${ }^{9}$ every downlink and twice before the suite of skydarks and skyflat observations. In addition to mitigating the persistent images, the frequent anneals also improve the stability of the bias on the arrays particularly at $5.8 \mu \mathrm{m}$. The anneal strategy evolved considerably during the first year of operations as ground tests did not indicate annealing was necessary.

\section{INTERPIXEL AND PHOTOMETRIC STABILITY}

The total gain of IRAC is determined by observing calibration stars of known fixed flux at specific locations on the imaging array, and that calibration is then propagated to all pixels on the array via a separate calibration of the pixel-wise relative gain response (flatfield). Below we show the stability of these two separate calibrations, which together determine the photometric calibration of IRAC.

The pixel-to-pixel relative responsivity of IRAC remains unchanged to within our ability to measure (which in all channels is less than a fraction of a percent) over the five year duration of the nominal mission. Figure 1 illustrates the fractional uncertainty in the individual measurements of the IRAC flat-field, which are repeated typically twice a month. Each individual flat-field was constructed from an object filtered median stack of observations of a high background. The flat-field applied to IRAC data is derived from these individual measurements. To within our measurement uncertainty, any individual flat-field does not vary from the median flat in a statistically significant way. The fluctuations seen as a function of time are attributable to variations in the sight-lines of the measurements relative to the Galactic and ecliptic planes.

The photometric stability of IRAC was monitored by trending aperture photometry of two of the primary IRAC calibrators (HD 165459 and NPM1+67.0536). The standard calibration observations at the beginning and end of each IRAC campaign were used. Each star is observed at 5 positions (center and centers of the four quadrants for each array) with sufficient integration time so that each measurement has a signal-to-noise in excess of 100 . Bad pixels and radhits were masked before performing aperture photometry using a two pixel radius aperture and 2-6 pixel background annulus. The array position-dependent photometric correction maps were applied as was the $3.6 \mu \mathrm{m}$ pixel-phase (intrapixel photometric variation) correction ${ }^{5}$. Figure 2 plots the scatter of the photometry of each star about the mean flux density as a function of time. The error bars are the formal measurement uncertainties $(1 \sigma \mathrm{rms})$ of each five position 
observation set and include both the Poisson contribution and readnoise. The peak-to-peak difference is $<3 \%$ for all four arrays. The rms scatter in the normalized photometry is $2.0 \%, 0.6 \%, 0.5 \%, 0.5 \%$ for the $3.6,4.5,5.8$ and $8.0 \mu \mathrm{m}$ arrays, respectively. The larger scatter at $3.6 \mu \mathrm{m}$ is due to residual of the pixel-phase effect and the use of a onedimensional (distance from center of pixel) functional fit. The scatter should be reduced with a two-dimension (dx, dy) functional fit $^{10}$. The slight offset in photometry $(\sim 2 \%)$ for $5.8 \mu \mathrm{m}$ at the beginning of the mission is due to a modification in the anneal strategy for IRAC. The $5.8 \mu \mathrm{m}$ array was not routinely annealed for the first 30 days of nominal operations.

\section{BIAS STABILITY}

Estimating the readnoise on orbit is difficult for IRAC as the shutter is not used in flight. Instead of true bias measurements, we create bias maps (skydarks) for each integration time using stacked observations of low background regions (near the North Ecliptic Pole). To measure the readnoise, we choose the shortest integration time skydarks (2 second frametime) to minimize the contribution from true signal.

For each of the skydarks across the first 45 campaigns, we calculated the standard deviation of a $50 \times 50$ box near the center of the array $(103: 153,103: 153)$ in electrons, and graph that versus the day since start of nominal operations (Figure 3). Bad/noisy pixels were masked using the standard bad pixel masks (pmasks) that are periodically generated. The standard deviation of the skydark should include contributions from the Poisson noise of the background, the read noise and clutter noise from unrejected, faint compact sources. The standard deviations for all channels are stable with time and in agreement with lab (pre-launch) measurements of readnoise and the expected noise contribution due to the background (mostly zodiacal light). The offset in the trends at 500 days is due to an increase in the number of frames used in later skydark observations. The number of pointings used was increased from nine to eighteen; the observed decrease in standard deviation is due to the $\sqrt{N}$ reduction in noise with increased number of samples. For the 3.6 and $4.5 \mu \mathrm{m}$ arrays the decrease in noise is almost exactly a factor of 1.4. The $5.8 \mu \mathrm{m}$ has slightly higher than expected noise which is likely due to residual pinstriping in the bias due to imperfect temporal/history dependent bias variation ${ }^{9}$ (first frame effect) removal. The $8.0 \mu \mathrm{m}$ array has some variations around the last part of 2006 (days 1000-1200). These variations are most likely due to a few unmasked noisy pixels as the last pmask update was in February 2006. The slightly sinusoidal trend at $8 \mu \mathrm{m}$ may be due to Spitzer's motion through the zodiacal dust cloud.

\section{BAD AND NOISY PIXEL TRENDING}

The bad and noisy pixel behavior of the arrays has been examined at several epochs during the mission, typically separated by 6-9 months (except for the case of the solar flare in January 2005, for which data from campaigns both prior to the flare and immediately following were examined). From the start of nominal operations, we annealed the 3.6 and $8.0 \mu \mathrm{m}$ arrays to mitigate long-term latents. After the first 30 days of nominal operations, the $5.8 \mu \mathrm{m}$ array was also routinely annealed as the anneals appeared to stabilize the array noise properties. After year 2, all arrays are annealed every 12-24 hours of IRAC operations.

In general, bad pixels tend to appear (presumably through radiation hits) and disappear (presumably through annealing) on timescales of days to months. For the long term trending described here and shown in Figure 4, we concentrated on pixels which were consistently bad over a several month interval. For each epoch, two skydark (100s frametime) observations and two skyflat (also 100s frametime) observations for the three campaigns approximately centered on the time in question were examined. Any pixel which was found to be more than twice as noisy as the median noise value in either the dark or flat data in all six dark or flat observations examined was flagged, and counted as noisy. Also flagged were any dead pixels (only seen at $3.6 \mu \mathrm{m}$ ), with a response of less than $1 \%$ of the median background value in the flats. The top two rows of the arrays were excluded from the analysis as, since launch, the top row for the 3.6, 4.5 and $8.0 \mu \mathrm{m}$ arrays, and the top two rows at $5.8 \mu \mathrm{m}$ have been consistently noisier than the bulk of the array.

The results indicate no significant change in the number of bad pixels since launch. In fact, the numbers seem to have decreased in some cases, perhaps because of improvements in annealing strategy. In particular, there has been no increase in the number of dead pixels at $3.6 \mu \mathrm{m}$, and no appearances of dead pixels in other channels, suggesting that 


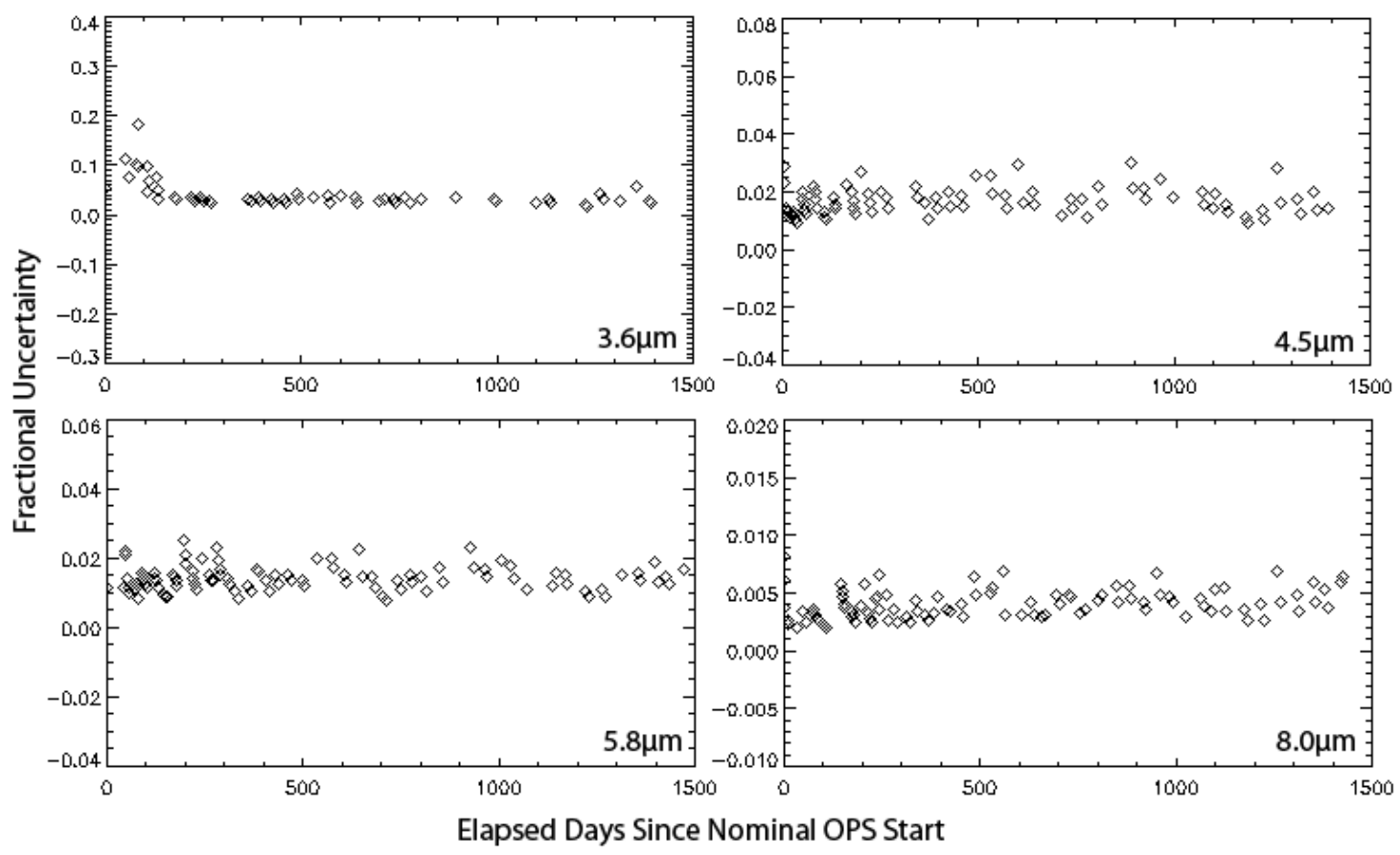

Figure 1. Variation in flat-field uncertainty since start of nominal operations. The variation in the uncertainty of the individual flat-field measurements is constant in time..

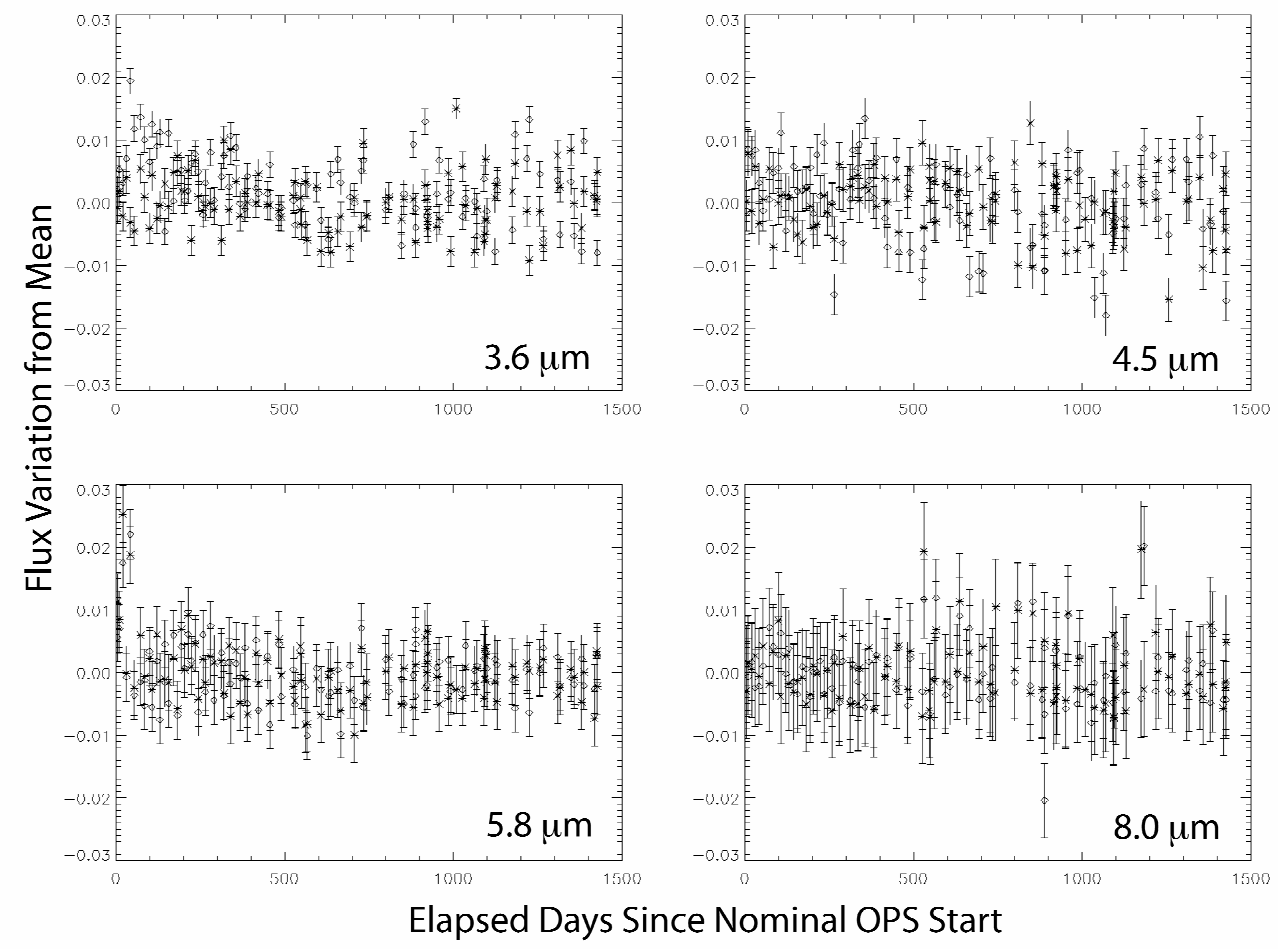

Figure 2. Temporal variation in IRAC photometry as determined for two calibrators, NPM1+67.0536 (asterisks) and HD 165459 (diamonds) for all four IRAC channels. The variation of each measurement about the mean measurement for each star is plotted versus time from start of nominal operations in days. 

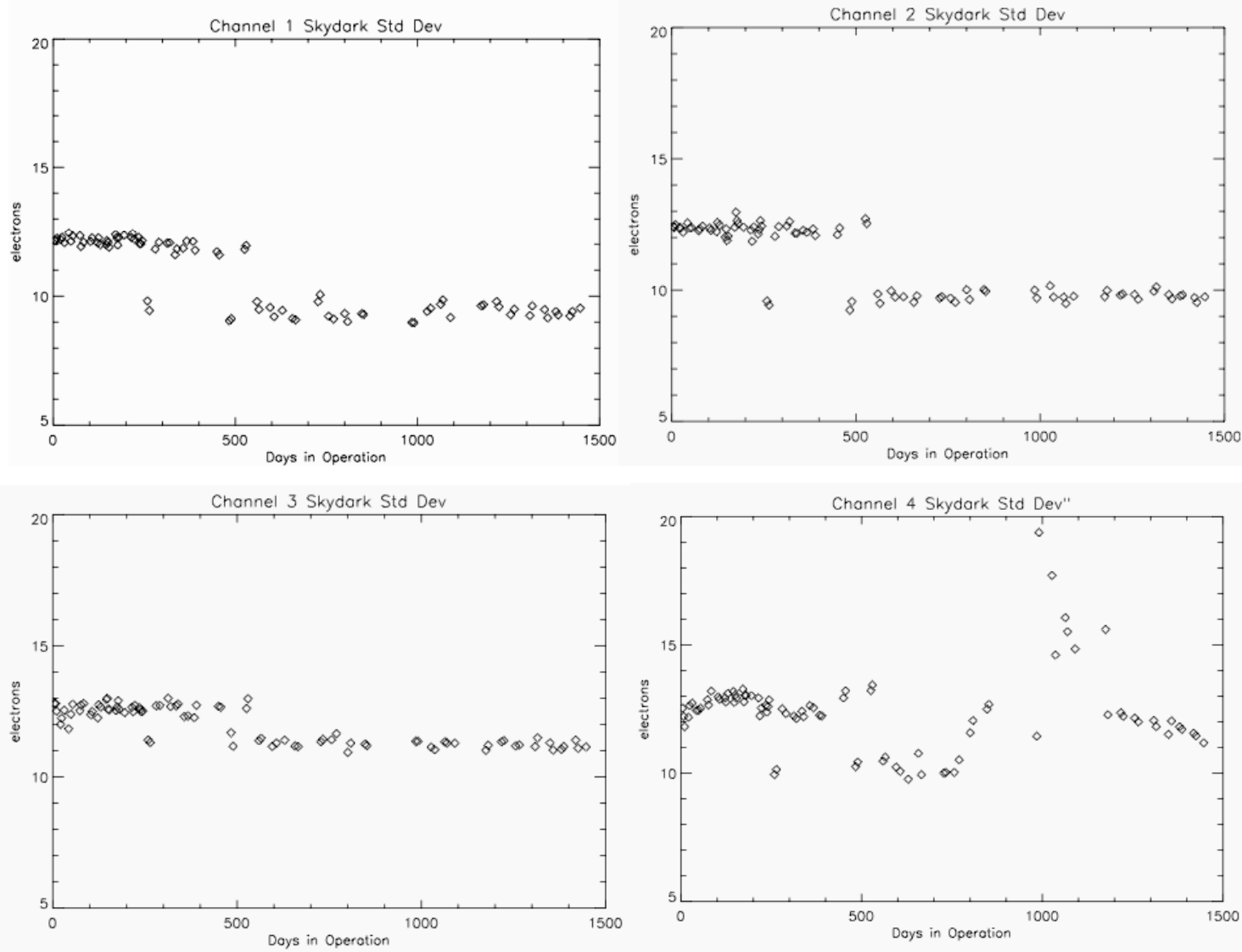

Figure 3. Standard deviation in electrons of a representative region of the bias calibration observations (skydarks) as a function of time since the start of nominal operations for the 3.6, 4.5, 5.8 and $8.0 \mu \mathrm{m}$ arrays, respectively.

severe radiation damage has not occurred. The number of noisy pixels at $8.0 \mu \mathrm{m}$ is increasing with time ${ }^{11}$, but remains a small fraction $(<<1 \%)$ of the entire array.

\section{SCIENCE ENABLED}

The high degree of stability achieved was not required in the design of IRAC although it is most certainly a result of the design and the high degree of thermal stability of the focal plane arrays. As a result of the stability and due to the ingenuity of the observing community, IRAC observations are able to investigate the nature of transiting exoplanets including the first definitive detection of water in an exoplanet atmosphere ${ }^{12}$ and a map of the longitudinal thermal profile of an exoplanet ${ }^{13}$. Observations of transiting hot Jupiters require photometric precisions of better than $0.5 \%$, secondary eclipse measurements of these same systems require precisions of order $0.1 \%$, while measurements of the thermal profile require precisions of $\sim 0.02 \%$. For studies of exoplanet atmospheres, high precision relative photometry between bandpasses is required; that is, the absolute calibration has to be stable modulo a systematic factor applied to all arrays equally. Similar measurements of Earth sized exoplanets would require two orders of magnitude better precision. It is possible though not yet confirmed that IRAC observations could detect the transit of planets of this size. The key question to answer is whether systematic sources of relative photometric error can be sufficiently trended so that the random component will go as the root of the number of observations of the transit.

High precision relative photometry observations with IRAC are best conducted by staring at the desired target for the desired duration. The noise of these observations (after accounting for systematics) is $\sim 130-140 \%$ of the Poisson noise of a given observation. Staring observations keep the target on the same set of pixels thereby minimizing fluctuations due 
to interpixel gain and bias variations. Unfortunately, the attitude for a staring observation is not strictly fixed with time as Spitzer exhibits a small ( $\sim .15$ arcsecond) periodic ( $\sim 3000$ seconds) pointing wobble ${ }^{14}$ believed to be due to thermal fluctuations between the star tracker and the telescope boresight. The wobble coupled with the pixel phase effect at 3.6 $\mu \mathrm{m}(4 \%$ peak to peak) and $4.5 \mu \mathrm{m}(<1 \%$ peak to peak $)$ is the dominant source of systematic error for precision
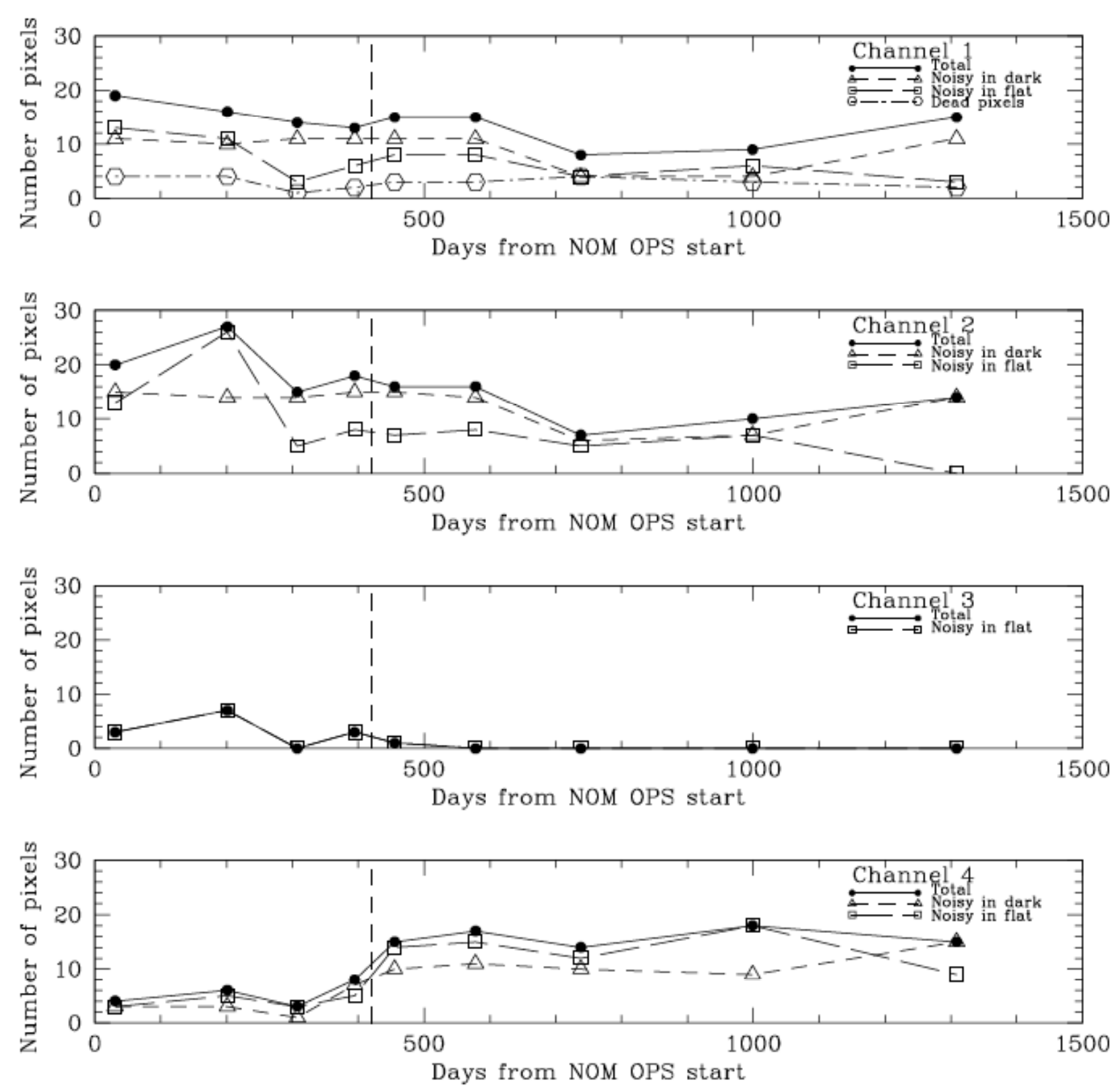

Figure 4. Bad and noisy pixels as a function of time. From top to bottom, the bad pixel time trends for 3.6, 4.5, 5.8 and 8.0 $\mu \mathrm{m}$ arrays. Pixels were classed as noisy in the dark, noisy in the flat, or dead ( $3.6 \mu \mathrm{m}$ array only). Note many pixels are noisy in both dark and flat. The dashed vertical line corresponds to the solar storm in January 2005.

photometry. As the photometric variations due to pixel phase are a function of source position relative to pixel center, the pixel phase effect can be removed by correlating flux variation with centroid position. As the effect is an intrinsic 
property of the detector, maps of the flux variation with pixel phase can be developed ${ }^{10}$. For the $8.0 \mu \mathrm{m}$ array, the most significant systematic is an increase in the measured flux of a source as a function of time. It has been suggested that this effect is due to charge trapping in the array and initial mitigation attempts by observing a bright region prior to the science observation to fill the traps appear to be effective ${ }^{15}$. Observing a bright patch of sky such that a fluence of $\sim 1 \times$ $10^{8} \mathrm{e}^{-} /$pixel is reached appears to almost entirely remove the observed flux ramping during a photometric monitoring observation. At $5.8 \mu \mathrm{m}$, there is a trend to lower measured flux with time during a staring observation. As the flux decreases with time, this phenomena cannot be due to charge trapping in the individual detectors. Due to the paucity of long-term photometric monitoring observations at $5.8 \mu \mathrm{m}$, there has been little study of the effect and there is no known mitigation strategy. For transit and eclipse observations, this effect can be removed as a linear trend ${ }^{16}$.

\section{WARM IRAC CAPABILITIES / STABILITY}

The cryogenic portion of the Spitzer mission is predicted to end in mid April 2009; however, as the telescope is also passively cooled, observations can be conducted with the InSb arrays after cryogen depletion. After cryogen is exhausted, the primary mirror temperature will increase to $26-29 \mathrm{~K}$ with the IRAC cold assembly being $\sim 1 \mathrm{~K}$ warmer. Ground tests ${ }^{17}$ of similar arrays in the flight mounts at the University of Rochester have shown that the IRAC InSb arrays should have similar performance characteristics at $30 \mathrm{~K}$ as they do at the current operating temperature of $15 \mathrm{~K}$. The focal plane heaters can heat the arrays to $40 \mathrm{~K}$; therefore, the arrays can be actively thermally controlled near the passively cooled instrument chamber temperature. Once the telescope reaches thermal equilibrium, which make take up to five weeks after cryogen exhaustion, the temperatures of the optics should be as stable as they are now. Based on data from the initial cooldown of Spitzer, there should be no measurable change in the optical properties during a warm-up to $29 \mathrm{~K}$. We reasonably expect the noise properties to be approximately the same as nominal mission and have every expectation that the arrays will remain as stable as they are now. As the number of bad, noisy or hot pixels is not increasing significantly with time for any array but in particular the 3.6 and $4.5 \mu \mathrm{m}$ arrays, it is expected that the arrays will maintain their high level of operable pixels. Currently, a two year warm mission ${ }^{18}$ is being planned with a call for large (1000 hour) 'exploration' class legacy proposals to be released in July 2008.

\section{CONCLUSIONS}

We have trended the stability of the four IRAC arrays over the course of the first four years of the Spitzer mission. To within our measurement uncertainty, the performance (gain, read noise, photometric stability) of the IRAC arrays has not changed with time. We note a slight increase in the number of hot pixels in the $8.0 \mu \mathrm{m}$ array as a function of time. The number of operable pixels at $8.0 \mu \mathrm{m}$ is still well within specification and will remain so throughout the cryogenic lifetime of Spitzer. The remaining arrays show no increase in the number of bad and/or hot pixels. It is reasonable to conclude that the 3.6 and $4.5 \mu \mathrm{m}$ arrays should remain stable during the post-cryogenic phase of the Spitzer mission.

We examined four aspects of IRAC calibration as a function of time. The variation in pixel response as a function of time was trended using our dedicated flat-field observations. The photometric stability was measured from repeated observations of two of the primary calibrators for IRAC. Variations in readnoise were estimated using the bias calibration observations of a low background region (skydarks) using a short integration time. Identification of hot and noisy pixels was performed by analyzing temporal variations in long integration time skydarks.

The authors acknowledge the efforts of our colleagues in the IRAC instrument and instrument support teams in maintaining and characterizing the IRAC instrument. This work is based on observations made with the Spitzer Space Telescope, which is operated by the Jet Propulsion Laboratory, California Institute of Technology under a contract with NASA. Support for this work was provided by NASA through an award issued by JPL/Caltech. 


\section{REFERENCES}

[1] Fazio, G. G., Hora, J. L., Allen, L. E., Ashby, M. L. N., Barmby, P., Deutsch, L. K., Huang, J.-S., et al., “The Infrared Array Camera (IRAC) for the Spitzer Space Telescope “, ApJS, 154, 10-17, (2004).

[2] Hora, J. L., Fazio, G. G., Allen, L. E., Ashby, M. L. N., Barmby, P., Deutsch, L. K., Huang, J.-S., et al., "In-flight performance and calibration of the Infrared Array Camera (IRAC) for the Spitzer Space Telescope “, Proc. SPIE 5487, 77-92, (2004).

[3] Werner, M. N., Roellig, T. L., Low, F. J., Rieke, G. H., Rieke, M., Hoffmann, W. F., Young, E., et al., “The Spitzer Space Telescope Mission", ApJS, 154, 1-9, (2004).

[4] Carey, S. J., Lacy, M. D., Laine, S. J., Reach, W. T., Surace, J. A., Glaccum, W. J., Hora, J. L., “Observing with the infrared array camera (IRAC) on the Spitzer Space Telescope”, Proc. SPIE 5487, 211-222, (2004).

[5] Reach, W. T., Megeath, S. T., Cohen, M., Hora, J., Carey, S., Surace, J., Willner, S. P., Barmby, P., et al., “Absolute Calibration of the Infrared Array Camera on the Spitzer Space Telescope", PASP, 117, 978-990, (2005).

[6] Benjamin, R. A., Churchwell, E., Babler, B. L., Bania, T. M., Clemens, D. P., Cohen, M., Dickey, J. M., et al., "GLIMPSE. I. An SIRTF Legacy Project to Map the Inner Galaxy”, PASP, 115, 953-964, (2003).

[7] Egami, E., Kneib, J.-P., Rieke, G. H., Ellis, R. S., Richard, J., Rigby, J., Papovich, C., et al., "Spitzer and Hubble Space Telescope Constraints on the Physical Properties of the z 7 Galaxy Strongly Lensed by A2218”, ApJL, 618, L5-L8, (2005).

[8] Charbonneau, D., Allen, L. E., Megeath, S. T., Torres, G., Alonso, R., Brown, T. M., Gilliland, R. L. et al., "Detection of Thermal Emission from an Extrasolar Planet", ApJ, 626, 523-529, (2006).

[9] Glaccum, W., Willner, S. P., Surace, J. A., Hora, J. T., Reach, W. T., Lowrance, P., Carey, S. J., et al., "IRAC InSb and Si:As arrays: anneals, image persistence, and hysteresis effects in dark images", (this proceeding).

[10] Mighell, K. J., Glaccum, W., Hoffmann, W., "Improving the photometric precision of IRAC Channel 1", (this proceeding).

[11] Hora, J. L., Patten, B. M., Fazio, G. G., Glaccum, William J., "The effects of cosmic rays and solar flares on the IRAC detectors: the first two years of in-flight operation", Proc. SPIE 6276, 62760J, (2006).

[12] Tinetti, G.; Vidal-Madjar, A., Liang, M.-C., Beaulieu, J.-P., Yung, Y., Carey, Sean; Barber, R. J., et al., "Water vapour in the atmosphere of a transiting extrasolar planet", Nature, 448, 169-171, (2007).

[13] Knutson, H. A., Charbonneau, D., Allen, L. E., Fortney, J. J., Agol, E., Cowan, N. B., Showman, A. P., et al. , “A map of the day-night contrast of the extrasolar planet HD 189733b" 2007, Nature, 447, 183-186, (2007).

[14] Morales-Calderón, M., Stauffer, J. R., Kirkpatrick, J. D., Carey, S.; Gelino, C. R.; Barrado y Navascués, D.; Rebull, L., "A Sensitive Search for Variability in Late L Dwarfs: The Quest for Weather”, ApJ, 653, 1454-1463, (2006).

[15] Knutson, H., Deming, D., private communtication (2008).

[16] Beaulieu, J.-P., Carey, S., Ribas, I., Tinetti, G., "Primary Transit of the Planet HD 189733 b at 3.6 and 5.8 $\mu \mathrm{m}$ ", ApJ, 677, 1343-1347, (2007).

[17] McMurty, C. W., Pipher, J. L., Forrest, W. J., "Spitzer space telescope: dark current and total noise prediction for InSb detector arrays in the infrared array camera (IRAC) for the post-cryogen era", Proc. SPIE 6265, 08, (2006).

[18] Storrie-Lombardi, L. J., et al., [The Science Opportunities of the Warm Spitzer Mission], AIP, 943, (2007). 